\title{
Use of near infrared spectroscopy for hemodynamic monitoring in pediatrics
}

\author{
Diego A. Brandoni ${ }^{a} \mathbb{D}$, M. Paula Martínez Da Bove ${ }^{a} \mathbb{D}$, Guillermo E. Moreno $^{a}(\mathbb{D}$
}

\begin{abstract}
Near infrared spectroscopy (NIRS) is a noninvasive optical technique for the evaluation of regional tissue oxygenation using transcutaneous detectors. In recent years, publications about this topic have increased exponentially; this reflects the growing interest among investigators and clinicians about this new technology and its potential benefits for pediatric patients. The objective of this review is to know the functioning and potential uses of regional saturation measured by NIRS and establish future challenges.

Key words: NIR spectroscopy, transcutaneous oximetry, cardiac surgical procedures, hemodynamic monitoring, low cardiac output.
\end{abstract}

http: / / dx.doi.org/ 10.5546/ aap.2022.eng.129

To cite: Brandoni DA, Martínez Da Bove MP, Moreno GE. Use of near infrared spectroscopy for hemodynamic monitoring in pediatrics. Arch Argent Pediatr 2022;120(2):129-135.

a. Cardiovascular Care Unit (ICU 35), Hospital de Pediatría S.A.M.I.C. "Prof. Dr. Juan P. Garrahan," Autonomous City of Buenos Aires, Argentina.

E-mail address:

Diego A. Brandoni: diego.brandoni@gmail. com

Funding:

None.

Conflict of interest: None.

Received: 3-24-2021

Accepted: 9-9-2021 adult patients who underwent carotid a reduced oxygen saturation determined by NIRS and a slowed electroencephalogram activity in balance using experimental heart and brain infrared transillumination.

Ten years later, Ferrari et al., ${ }^{2}$ showed a correlation between

Technological advances allow to use new tools to monitor critically ill patients. This was a non-systematic review about one of them, near establish the basics of how it works and potential uses in pediatric

\section{ISTORY AND BEGINNING}

published in 1977 in Science. ${ }^{1}$ It allowed Frans Jöbsis to demonstrate the possibility of measuring changes in hemoglobin-oxyhemoglobin a endarterectomy.

Since the early 2000s, several studies have been published to validate the use of NIRS in pediatric patients: Nagdyman et al., ${ }^{3}$ studied 43 children after undergoing surgery for congenital heart disease to determine the relation between cerebral tissue oxygenation index (TOI) and central venous oxygen saturation $\left(\mathrm{SvO}_{2}\right)$. TOI describes the relation between oxygenated hemoglobin and total hemoglobin, as determined by NIRS. Measured $\mathrm{SvO}_{2}$ values revealed a significant correlation with the cerebral oxygenation index, with a Pearson correlation value of 0.52 and a $p$ value $<0.001$. In another prospective study, ${ }^{4}$ the same author compared the TOI value determined by NIRS with oxygen venous saturation measured at the jugular bulb in children and, again, demonstrated a significant correlation between both values with a high specificity $(91 \%)$, but a low sensitivity $(46 \%)$.

A correlation was also established between NIRS and plethysmography. A study conducted in 2007 showed an intraclass correlation of 0.93 for the NIRS as an indicator of distal perfusion. ${ }^{5}$ Another prior study demonstrated that forearm blood flow estimated by NIRS correlated to that estimated by plethysmography, with an $\mathrm{r}$ value $=0.853 \sim 0.981$ and $p$ value $<0.001 .^{6}$

Although NIRS was initially used as a surrogate for regional cerebral blood flow, its use has expanded to other applications, as described below.

\section{DEFINITION AND FUNCTIONING}

Oxygen saturation determined 
by NIRS is an optical technique that allows a continuous, non-invasive measurement in real time of the balance between regional oxygen availability $\left(\mathrm{DO}_{2}\right)$ and consumption $\left(\mathrm{VO}_{2}\right)$ (Figure 1), based on the principle that many substances absorb and disseminate infrared light in different levels at varying wavelengths.

An infrared spectrometer emits light towards a sample and measures the proportion of light reflected within a wide range of wavelengths. Using the modified Beer-Lambert law, it measures relative saturated and desaturated hemoglobin levels, resulting in an ongoing and non-invasive assessment of oxygen tissue saturation through a quantitative evaluation of hemoglobin color in the optical field below the detector.

Unlike pulse oximetry, NIRS devices reflect oxygen uptake in the tissue bed; this is known as regional oxygen saturation $\left(\mathrm{rSO}_{2}\right)$. In other words, they measure the balance between the oxygen delivered to the tissue and that obtained at this tissue level. The ongoing measurement of tissue oxygenation reflects the state of perfusion and allows to monitor variations in real time, to prevent shock. ${ }^{7-11}$
These values are obtained by placing a detector on the skin of the area where the measurement will be made: forehead (brain) (Figure 2), abdomen (mesentery), lumbar area (kidneys).

In general, 2 detectors are used to assess 2 different circulation models. Each detector has a source of light and 2 photodetectors. Photons emitted by the source of light are disseminated into the tissue bed. Those not absorbed, return to the skin photodetector. The oxygen saturation value determined by NIRS accounts for the amount of light absorbed in the tissue bed. Such value accounts for an average of arterial, venous, and capillary oxygenation; $75-80 \%$ corresponds to the venous portion. ${ }^{12}$

These measurements should be interpreted in the setting of the patient because a low value may be due to a higher oxygen extraction (as in the case of shock) or a reduced blood flow (ischemia) in said region, resulting in an alteration of tissue oxygen delivery.

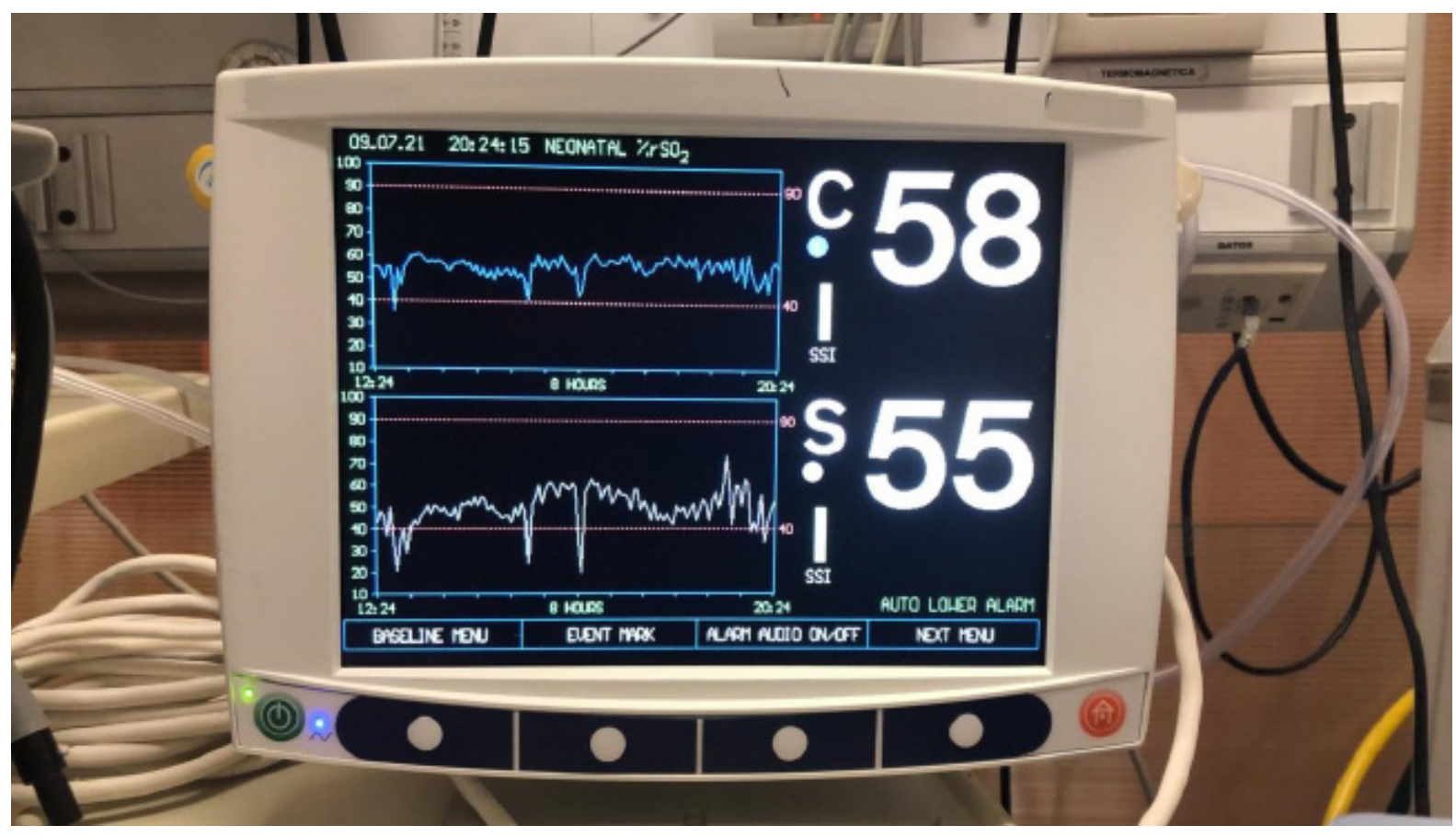




\section{NORMAL VALUES}

In 2001, Kurth et al., published a study that measured regional cerebral saturation $\left(\mathrm{ScO}_{2}\right)$ determined by NIRS in both pediatric patients with congenital heart disease before undergoing cardiovascular surgery and healthy individuals. They found that mean $\mathrm{ScO}_{2}$ was $68 \%$ (standard deviation $[\mathrm{SD}] \pm 10 \%$ ) in healthy children and in those with non-cyanotic heart defect. Patients with cyanotic heart defect (transposition of the great arteries, pulmonary atresia) had lower values: $47 \pm 11$ and $38 \pm 6$, respectively. ${ }^{13}$

Establishing normal values for somatic regional oxygen saturation $\left(\mathrm{SsO}_{2}\right)$ in the abdominal wall or the renal cell is harder; this depends on the patient's weight and age, the abdominal wall thickness, the type of detector used, and the site to be assessed. For example, $\mathrm{SsO}_{2}$ measured at the renal cell correlates with oxygen saturation in the renal vein and the inferior vena cava only in patients who weigh less than $10 \mathrm{~kg}$. Another study ${ }^{14}$ demonstrated an adequate correlation between $\mathrm{SsO}_{2}$ in the infraumbilical region with blood flow measured by Doppler in the superior mesenteric artery in neonates who did not have abdominal wall edema or ascites.

Due to such difficulty to standardize normal
$\mathrm{SsO}_{2}$ values, the difference in somatic-cerebral saturation, which ranges between $10 \%$ and $20 \%$ in healthy individuals, may be useful because $\mathrm{SsO}_{2}$ is generally higher due to oxygen extraction from cerebral flow. In a study published in 2010, Bernal et al., ${ }^{15}$ monitored healthy neonates using a NIRS device placed in the frontal region and the right side at the T2-L2 level and found an average somaticcerebral saturation difference of $8.9 \% \pm 9.4 \%$. In pathological situations where the blood flow is redistributed to preserve the cerebral bed, the difference is close to $0 \%$ or negative. Hanson et al., ${ }^{16}$ demonstrated that the difference in somatic-cerebral saturation in dehydrated patients admitted to the emergency department was low and increased with fluid resuscitation, which confirms a dose-response relation.

The implementation of oxygen saturation with multilevel NIRS provides simultaneous measurements of $\mathrm{ScO}_{2}$ and $\mathrm{SsO}_{2}$; this allows for an early detection of low cardiac output through changes in the somatic-cerebral difference.

\section{CURRENT USES \\ Cardiovascular surgery}

Pediatric cardiovascular surgery was one of the first disciplines that implemented the measurement of oxygen saturation using NIRS.

FIGURE 2. Cerebral regional oxygen saturation. Axial section obtained at the frontal level; the light travels in a curvilinear path across the underlying tissue and is absorbed by photodetectors

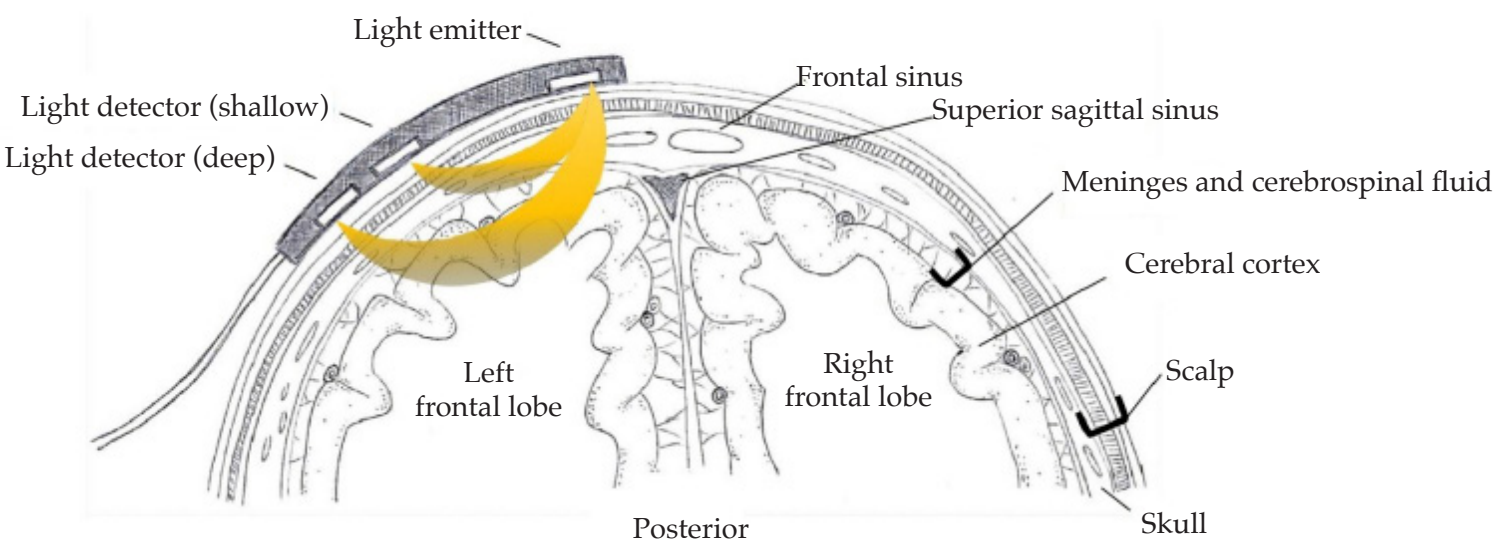

From Zaleski KL, Kussman BD. Near infrared spectroscopy in pediatric congenital heart disease, J Cardiothorac Vasc Anesth. 2020;34(2):489-500. 
Alterations in neurodevelopment are common among survivors of surgery for congenital heart disease which, even with a multifactorial etiology, may experience a partial improvement in prognosis, with an adequate neurological monitoring during surgery. ${ }^{17-21}$

Austin et al., ${ }^{17}$ were the first authors who reported improvements in neurological outcomes with the introduction of NIRS in a multimodal neurophysiological monitoring algorithm in patients undergoing extracorporeal circulation (ECC). In their 1997 study, they demonstrated that among patients who received an intervention based on the algorithm for the measurement of $\mathrm{ScO}_{2}$ by NIRS, cerebral blood flow measured by transcranial Doppler, and electroencephalogram monitoring, only $6 \%$ developed neurological sequelae (seizures and alterations in motor skills, vision or hearing loss) versus $26 \%$ in the group that did not follow the algorithm $(p<0.001)$.

However, there is still a debate about the clinical usefulness of NIRS in the perioperative period of cardiovascular surgery; further randomized clinical trials are required in the pediatric population to establish a coherent association between low cerebral NIRS values and poor neurological outcomes. ${ }^{18}$

Prospective observational studies have assessed the relationship between cerebral and somatic NIRS and other anaerobic metabolism indicators. In a series of 20 cases, ${ }^{19} \mathrm{SsO}_{2}$ measured at the posterior-lateral region and the anterior abdominal region showed a strong correlation with central venous oximetry and lactate values in the 48 hours following surgery. In a similar study of 23 infants, regional oxygen saturation $\left(\mathrm{rSO}_{2}\right)$ was measured at the level of the brain, viscera, kidneys, and muscles every 30 seconds, and the values at each site were compared to plasma lactate levels in the first 24 hours following surgery. A stronger correlation was observed between $\mathrm{ScO}_{2}$ determined by NIRS and lactate levels. In this series, $\mathrm{ScO}_{2}$ values below $65 \%$ in acyanotic children predicted a lactate level above $3 \mathrm{mmol} / \mathrm{L}$, with a $95 \%$ sensitivity and a $83 \%$ specificity. ${ }^{7,20}$

In a series of 79 patients with hypoplastic left heart syndrome (HLHS) who underwent surgery for stage 1 single ventricle, $\mathrm{SsO}_{2}$ below $60 \%$ and a difference between $\mathrm{ScO}_{2}$ and $\mathrm{SsO}_{2}$ close to 0 correlated to shock, post-operative complications, and a longer length of stay in the intensive care unit (ICU), with a $p<0.01$. $^{21}$

In 2016, Hickok et al., ${ }^{22}$ published an observational, prospective study that analyzed the relation between a low oxygen saturation determined by NIRS and the development of low cardiac output syndrome (LCOS) in 27 neonates with congenital heart disease who underwent heart surgery. Of them, $11(40 \%)$ developed LCOS, defined as a $\mathrm{pH}$ level below 7.3, lactate level higher than $4 \mathrm{mmol} / \mathrm{L}$ or the need to add a new vasoactive agent to the treatment. They obtained a consistent $\mathrm{SsO}_{2}$ determined by NIRS below $58 \%$, with a $100 \%$ sensitivity and a $69 \%$ specificity to detect LCOS, whereas a variability in NIRS values below $0.8 \%$ showed a $55 \%$ sensitivity and a $94 \%$ specificity to predict the development of LCOS. In the group of patients diagnosed with $\mathrm{LCOS}$, mean $\mathrm{ScO}_{2}$ and $\mathrm{SsO}_{2}$ in the renal cell were $48 \%$ and $50 \%$, respectively, which is consistent with the data obtained in other studies about the correlation of a difference of less than $10 \%$ between cerebral and somatic NIRS as a predictor of LCOS.

A study with a larger number of patients $(\mathrm{n}=329)$ published by Hoffman et al., ${ }^{23}$ in 2017 concluded that NIRS measurements during the postoperative period of congenital heart disease in neonates allowed to detect regional hypoxia-ischemia, even in the absence of an overall $\mathrm{SvO}_{2}$ reduction. In addition, regional saturation determined by NIRS was the best noninvasive predictor of extracorporeal membrane oxygenation (ECMO) in the first 6 hours following surgery, with an area under the ROC curve of 0.716 .

\section{Necrotizing enterocolitis}

Necrotizing enterocolitis (NEC) is the most severe gastrointestinal disease in neonates. It is associated with harmful effects in the short and long term, including high rates of mortality and neurodevelopmental impairment. The pathophysiology of NEC has not been completely elucidated yet; it is believed that the mechanism involves a complex interaction among several factors: neonatal gut mucosa immaturity, mesenteric ischemia, tissue hypoxia, enteral feeding, and the presence of infectious agents or toxins. NEC is more common among preterm infants; however, approximately $10 \%$ occurs in term or late preterm infants. These patients have additional risk factors that predispose them to intestinal ischemia, including congenital heart disease, intrauterine growth restriction or neonatal asphyxia.

McElhinney et al. ${ }^{25}$ found that patients with HLHS, univentricular heart with aortic 
arc interruption, truncus arteriosus, or aortopulmonary window had significantly higher rates of NEC than those with other types of heart disease.

These findings are consistent with those reported by De Witt et al., ${ }^{26}$ in a study of 2014, which compared the risk for NEC in patients with biventricular (30 patients) and univentricular (34 patients) morphology. Of them, 11 developed NEC, all in the univentricular group. Patients with NEC diagnosis had a lower visceral $\mathrm{rSO}_{2}$ $(32.6 \%$ versus $47.0 \%, p=0.05)$ and a higher rate of time with a $\mathrm{SsO}_{2}$ below $30 \%(48.8 \%$ versus $6.7 \%$, $p=0.04)$ than in the NEC group. This shows a difference in regional oxygenation determined by NIRS among patients with NEC.

\section{Extracorporeal circulation}

The use of oxygen saturation determined by NIRS has increased as hemodynamic monitoring in patients undergoing heart surgery with extracorporeal circulation (ECC) requirement. In an international survey administered to perfusionists, ${ }^{18}$ the use of $\mathrm{ScO}_{2}$ determined by NIRS during extracorporeal circulation was $74 \%$. Another survey, carried out in the United States, reported that $97 \%$ of facilities used cerebral regional oximetry during the Norwood procedure for HLHS. ${ }^{18}$

A study conducted by Haydin et al., ${ }^{27}$ compared cerebral oxygen saturation determined by NIRS, mean blood pressure (MBP), lactate levels, and pump flow during pediatric cardiovascular surgery using ECC and developed hypothermia. For this comparison, pump time was divided into 5 stages: 1) at the beginning of ECC, 2) cooling at $32{ }^{\circ} \mathrm{C}, 3$ ) at final target temperature, 4) rewarming at $32{ }^{\circ} \mathrm{C}$, and 5) before end of ECC. $\mathrm{ScO}_{2}$ values showed changes among the 5 studied stages; there was a significant increase during the cooling period, compared to stage $1(p<0.05)$. There was no significant change during the cooling period for the other analyzed parameters. Those investigators concluded that cerebral oxygen saturation determined by NIRS is effective to safely monitor a reduction in oxygen consumption and the corresponding cerebral protection.

\section{Extracorporeal membrane oxygenation}

In relation to the use of $\mathrm{ScO}_{2}$ determined by NIRS during ECMO, in 2020 Tsou et al., ${ }^{28}$ published an observational retrospective cohort study that analyzed 156 admissions to ECMO between 2008 and 2014. The final outcome was to determine patients' neurological status at discharge using the Pediatric Cerebral Performance Category. The authors found that the proportion of patients with a $\mathrm{ScO}_{2}$ value below $40 \%$ or a drop higher than $20 \%$ from baseline $\mathrm{ScO}_{2}$ was significantly higher in the group with unfavorable neurological outcomes at discharge. These values were associated with both mortality prior to discharge and pathological findings in images of the brain (stroke and overall injury post-asphyxia).

Based on these articles, NIRS use during ECMO may help to identify patients at risk for death or unfavorable neurological outcomes, and this may allow to implement early therapeutic measures.

\section{OTHER POTENTIAL USES \\ Early shock recognition}

Shock in pediatric patients results in a high mortality and morbidity rate worldwide. Its early detection and management are critical to reduce it. Unlike adult patients, arterial hypotension is a delayed sign of shock in children. ${ }^{29}$

Pichler et al., ${ }^{30}$ compared hypotension events in 2 groups of neonates (NIRS versus control). In the NIRS group, the difference between cerebral and somatic $\mathrm{rSO}_{2}$ was measured every 6 hours. If such difference was higher than $5 \%$, it was considered an early sign of centralized circulation (shock initiation) and actions were implemented to optimize perfusion (volume, inotropes). In the control group, oxygen saturation values determined by NIRS were not visible for the treating team, so the standard plan implemented at the unit was followed. The authors of this study concluded that using the difference between cerebral and somatic $\mathrm{rSO}_{2}$ values determined by NIRS may favor the early detection of alterations in microcirculation before they become clinically evident.

\section{Monitoring of peripheral perfusion}

Reports $^{31,32}$ have been recently published about the use of oxygen saturation determined by NIRS to monitor peripheral perfusion after lower extremity trauma or after femoral artery cannulation for ECC.

In 2019, Herbet et al., ${ }^{33}$ carried out a study to assess the use of $\mathrm{SrO}_{2}$ determined by NIRS to establish the risk for arterial occlusion following cardiac catheterization in 152 patients. $\mathrm{SrO}_{2}$ values were obtained by NIRS placing detectors 
bilaterally, in the posterior and lateral area of both calves. A significant reduction in regional oxygen saturation was observed in the extremity that underwent the procedure compared to the opposite extremity. $\mathrm{SrO}_{2}$ values determined by NIRS did not show a correlation with the subjective assessment of peripheral perfusion. One patient had a complication of missed heartbeat, which was managed with heparin; this patient showed a clear reduction in regional saturation, with a progressive improvement after treatment initiation.

\section{Neurosurgery}

There are currently no non-invasive methods to measure intracranial pressure. Lang et al., ${ }^{34}$ proposed using $\mathrm{ScO}_{2}$ determined by NIRS to assess cerebral perfusion in children with hydrocephalus and intracranial hypertension who were scheduled for surgery. They compared $\mathrm{ScO}_{2}$ before and after cerebrospinal fluid shunt in 22 patients. A significant difference was observed in $\mathrm{ScO}_{2}$ values determined by NIRS; this suggests an improvement in cerebral oxygenation, which correlated to the resolution of intracranial hypertension symptoms following recovery from anesthesia.

\section{Transfusions}

It is known that absolute and isolated hematocrit and hemoglobin values should not be the only parameters to be taken into consideration when indicating red blood cell transfusions. A potential strategy to guide transfusion support may be fractional tissue oxygen extraction (FTOE) as a surrogate measure for the cerebral oxygen supply-demand ratio. This indicator results from the ratio between arterial oxygen saturation and regional oxygen saturation determined by NIRS. Neunhoeffer et al., ${ }^{35}$ proposed using a high FTOE as a marker for transfusion requirement. They included 62 neonates and infants younger than 6 months who underwent a major surgery (heart disease, diaphragmatic hernia, and omphalocele). They found a clear improvement in cerebral regional oxygen saturation following transfusion, especially among patients with a cerebral FTOE higher than 0.4 .

\section{Transplantation}

A 2021 systematic review ${ }^{36}$ found that oxygen saturation determined by NIRS may also be useful in liver and kidney transplantation. They analyzed 5 relevant publications: 3 studied graft perfusion in kidney transplantation and 2 referred to liver transplantation. Their main finding was that a drop in regional saturation predicted vascular graft complications.

\section{FINAL COMMENT}

Oxygen saturation determined by NIRS is a monitoring method that allows for a non-invasive measurement in real time of the balance between regional oxygen availability and consumption. It provides continuous information about changes in cerebral and systemic flow and allows for the early detection of negative clinical events.

In recent years, its varied uses have increased not only in the operating room, but also in pediatric and neonatal intensive care units.

The accuracy of oxygen saturation determined by NIRS may be compared with invasive measurements, such as $\mathrm{SvcO}_{2}$, serves as an excellent complement to $\mathrm{SvcO}_{2}$ and is very useful to monitor cerebral perfusion during cardiovascular surgery.

An absolute $\mathrm{ScO}_{2}$ value below 50 or a $20 \%$ drop from baseline, as determined by NIRS, calls for a procedure to rule out ventilatory disorders (hypocapnia) or events that increase the cerebral metabolic demand (such as seizures or hyperthermia), optimize analgosedation, and monitor $\mathrm{SvO}_{2}$ and hematocrit levels.

The use of oxygen saturation determined by NIRS in older patients is limited. Most studies have focused on infants and neonates. Another barrier in the standardization of NIRS use is the large inter- and intra-individual variability of measurements; this hurdles the possibility of obtaining normal values and cutoff points. ${ }^{37}$

Further randomized controlled trials are required in the pediatric population to demonstrate that the use of oxygen saturation determined by NIRS can serve as a routine hemodynamic monitoring strategy to reduce mortality or the length of stay and to warrant the inherent cost of any new technology implementation.

Understanding both how available devices work and their limitations will allow $\mathrm{SrO}_{2}$ determined by NIRS to become, in the setting of blood flow monitoring, the equivalent of saturometers for respiratory monitoring: a tool that provides continuous and non-invasive information that may significantly increase the detection of an imbalance between oxygen supply and demand at the level of microcirculation. 


\section{REFERENCES}

1. Jöbsis FF. Noninvasive, infrared monitoring of cerebral and myocardial oxygen sufficiency and circulatory parameters. Science. 1977; 198(4323):1264-7.

2. Ferrari M, Zanette E, Sideri G, Giannini I, et al. Effects of carotid compression, as assessed by near infrared spectroscopy, upon cerebral blood volume and hemoglobin oxygen saturation. J R Soc Med. 1987; 80(2):83-7.

3. Nagdyman N, Fleck T, Barth S, Abdul-Kahliq H, et al. Relation of cerebral tissue oxygenation index to central venous oxygen saturation in children. Intensive Care Med. 2004; 30(3):468-71.

4. Nagdyman N, Fleck T, Schubert S, Ewert P, et al. Comparison between cerebral tissue oxygenation index measured by near-infrared spectroscopy and venous jugular bulb saturation in children. Intensive Care Med. 2005; 31(6):846-50.

5. Harel F, Denault A, Ngo Q, Dupuis J, Khairy P. Nearinfrared spectroscopy to monitor peripheral blood flow perfusion. J Clin Monit Comput. 2008; 22(1):37-43.

6. Homma S, Eda H, Ogasawara S, Kagaya A. Near-infrared estimation of $\mathrm{O}_{2}$ supply and consumption in forearm muscles working at varying intensity. J Appl Physiol (1985). 1996; 80(4):1279-84.

7. Ghanayem N, Hoffman G. Near Infrared Spectroscopy as a Hemodynamic Monitor in Critical Illness. Pediatr Crit Care Med. 2016; 17(8 Suppl 1):S201-6.

8. Marrin T, Moore J. Understanding near-infrared spectroscopy. Adv Neonat Care. 2011; 11(6):382-8.

9. McNeill S, Gatenby JC, McElroy S, Engelhardt B. Normal cerebral, renal and abdominal regional oxygen saturations using near-infrared spectroscopy in preterm infants. J Perinatol. 2011; 31(1):51-7.

10. Tobias JD. Cerebral oximetry monitoring with near infrared spectroscopy detects alterations in oxygenation before pulse oximetry. J Intens Care Med. 2008; 23(6):384-8.

11. Watzman HM, Kurth CD, Montenegro LM, Rome J, et al. Arterial and venous contributions to near-infrared cerebral oximetry. Anesthesiology. 2000; 93(4):947-53.

12. Weiss M, Dullenkopf A, Kolarova A, Schulz G, et al. Nearinfrared spectroscopic cerebral oxygenation reading in neonates and infants is associated with central venous oxygen saturation. Paediatr Anaesth. 2005; 15(2):102-9.

13. Kurth CD, Steven JL, Montenegro LM, Watzman HM, et al. Cerebral oxygen saturation before congenital heart surgery. Ann Thorac Surg. 2001; 72(1):187-92.

14. Gillam-Krakauer M, Cochran CM, Slaughter JC, Polavarapu $\mathrm{S}$, et al. Correlation of abdominal $\mathrm{rSO}_{2}$ with superior mesenteric artery velocities in preterm infants. J Perinatol. 2013; 33(8):609-612.

15. Bernal NP, Hoffman GM, Ghanayem NS, Arca MJ. Cerebral and somatic near-infrared spectroscopy in normal newborns. J Pediatr Surg. 2010; 45(6):1306-10.

16. Hanson SJ, Berens RJ, Havens PL, Kim MK, Hoffman GM. Effect of volume resuscitation on regional perfusion in dehydrated pediatric patients as measured by twosite near-infrared spectroscopy. Pediatr Emerg Care. 2009; 25(3):150-3.

17. Austin EH 3rd, Edmonds HL Jr, Auden SM, Seremet $\mathrm{V}$, et al. Benefit of neurophysiologic monitoring for pediatric cardiac surgery. J Thorac Cardiovasc Surg. 1997; 114(5):707-15; discussion 716.

18. Zaleski KL, Kussman BD. Near-Infrared Spectroscopy in Pediatric Congenital Heart Disease. J Cardiothorac Vasc Anesth. 2020; 34(2):489-500.

19. Kaufman J, Almodovar MC, ZukJ, Friesen RH. Correlation of abdominal site near-infrared spectroscopy with gastric tonometry in infants following surgery for congenital heart disease. Pediatr Crit Care Med. 2008; 9(1):62-8.

20. Chakravarti SB, Mittnacht AJ, Katz JC, Nguyen K, Joashi U. Multisite near-infrared spectroscopy predicts elevated blood lactate level in children after cardiac surgery. $J$
Cardiothorac Vasc Anesth. 2009; 23(5):663-7.

21. Hoffman GM, Ghanayem NS, Mussatto KM, Berens RJ, Tweddell JS. Postoperative two-site NIRS predicts complications and mortality after stage one palliation of HLHS. Anesthesiology. 2007; 107:A234.

22. Hickok RL, Spaeder MC, Berger JT, Schuette JJ, Klugman D. Postoperative abdominal NIRS values predict low cardiac output syndrome in neonates. World J Pediatr Congenit Heart Surg. 2016; 7(2):180-4.

23. Hoffman GM, Ghanayem NS, Scott JP, Tweddell JS, et al. Postoperative Cerebral and Somatic Near-Infrared Spectroscopy Saturations and Outcome in Hypoplastic Left Heart Syndrome. Ann Thorac Surg. 2017; 103(5):152735.

24. Schat TE, Schurink M, van der Laan ME, Hulscher JBF, et al. Near-Infrared Spectroscopy to Predict the Course of Necrotizing Enterocolitis. PLoS One. 2016; 11(5):e0154710.

25. McElhinney DB, Hedrick HL, Bush DM, Pereira GR, et al. Necrotizing enterocolitis in neonates with congenital heart disease: risk factors and outcomes. Pediatrics. 2000; 106(5):1080-7.

26. DeWitt AG, Charpie JR, Donohue JE, Yu S, Owens GE. Splanchnic near-infrared spectroscopy and risk of necrotizing enterocolitis after neonatal heart surgery. Pediatr Cardiol. 2014; 35(7):1286-94.

27. Haydin S, Onan B, Onan IS, Ozturk E, et al. Cerebral perfusion during cardiopulmonary bypass in children: correlations between near-infrared spectroscopy, temperature, lactate, pump flow, and blood pressure. Artif Organs. 2012; 37(1):87-91.

28. Tsou PY, Garcia AV, Yiu A, Vaidya DM, Bembea MM. Association of Cerebral Oximetry with Outcomes after ExtracorporealMembraneOxygenation. Neurocrit Care.2020; 33(2):429-37.

29. Mendelson J. Emergency Department Management of Pediatric Shock. Emerg Med Clin North Am. 2018; 36(2):42740.

30. Pichler G, Höller N, Baik-Schneditz N, Schwaberger B, et al. Avoiding Arterial Hypotension in Preterm Neonates (AHIP)-A Single Center Randomised Controlled Study Investigating Simultaneous Near Infrared Spectroscopy Measurements of Cerebral and Peripheral Regional Tissue Oxygenation and Dedicated Interventions. Front Pediatr. 2018; 6:15.

31. Schachner T, Bonaros N, Bonatti J, Kolbitsch C. Near infrared spectroscopy for controlling the quality of distal leg perfusion in remote access cardiopulmonary bypass. Eur J Cardiothorac Surg. 2008; 34(6):1253-4.

32. Vida VL, Padalino MA, Boccuzzo G, Stellin G. Nearinfrared spectroscopy for monitoring leg perfusion during minimally invasive surgery for patients with congenital heart defects. J Thorac Cardiovasc Surg. 2012; 143(3):756-7.

33. Herbert CE, Leshko J, Morelli D, Amankwah E, et al. Use of Near-Infrared Spectroscopy to Monitor Lower Extremity Perfusion in Pediatric Patients Undergoing Cardiac Catheterization. Pediatr Cardiol. 2019; 40(7):1523-9.

34. Lang S, Khanna O, Atkin NJ, Palma JE, et al. Perioperative near-infrared spectroscopy cerebral oxygen saturation in symptomatic pediatric hydrocephalus patients at risk for intracranial hypertension. J Neurosurg Pediatr. 2019; 1-7.

35. Neunhoeffer F, Hofbeck M, Schuhmann MU, Fuchs J, et al. Cerebral Oxygen Metabolism Before and After RBC Transfusion in Infants Following Major Surgical Procedures. Pediatr Crit Care Med. 2018; 19(4):318-27.

36. Ghidini F, Benetti E, Zucchetta P, Amigoni A, et al. Transcutaneous near-infrared spectroscopy (NIRS) for monitoring kidney and liver allograft perfusion. Int J Clin Pract. 2021; 75(5):e14034.

37. Green MS, Sehgal S, Tariq R. Near-Infrared Spectroscopy: The New Must Have Tool in the Intensive Care Unit? Semin Cardiothorac Vasc Anesth. 2016; 20(3):213-24. 